\title{
Comparative Study on Bone Mineral Densitometry in People Living with HIV with or without Highly Active Antiretroviral Therapy
}

\author{
Tutan Das ${ }^{1}$ Bhagyabati Devi² Ningthoukhongjam Reema ${ }^{2}$ Thangjam Gautam Singh ${ }^{3}$ \\ ${ }^{1}$ Department of Medicine, Regional Institute of Medical Sciences, \\ Imphal, India \\ 2Department of Medicine, Regional Institute of Medical Sciences, \\ Imphal, India \\ ${ }^{3}$ Department of Radiodiagnosis, Shija Hospital, Imphal, India

\begin{abstract}
Address for correspondence Ningthoukhongjam Reema, MBBS, MD, PGDGM, CCEBDM, Department of Medicine, Regional Institute of Medical Sciences, Lamphelpat, Imphal 795004, Manipur, India (e-mail: thangjamreema@gmail.com).
\end{abstract}

Int J Recent Surg Med Sci 2020;6:18-23

\begin{abstract}
Keywords

- bone mineral density

- DEXA

- HIV

- osteoporosis

Introduction Human immunodeficiency virus (HIV) is a disease that affects millions of people globally and affects almost all the body systems including bone metabolism. Derangement of bone mineral density (BMD) in HIV patients is well established in international literature but least studied in India. Therefore, this study aims to determine the association between BMD change and HIV infection with or without antiretroviral therapy (ART) and compare the different regimens of ART.

Materials and Methods The cross-sectional study was conducted at the Department of Medicine and ART Center of Regional Institute of Medical Sciences, Imphal, India. A total of $50 \mathrm{HIV}$ patients were screened by a central dual-energy X-ray absorptiometry (DEXA) examination for measuring BMD. Correlation of BMD with a CD4 count, and different ART regimens were also studied.

Results In our study, majority of the patients (29 [58\%]) had low BMD. Of the 29 patients, 18 (36\%) had osteopenia and $11(22 \%)$ had osteoporosis. Of the ART naive patients, $81.8 \%$ have reduced BMD. Among different ART regimens, tenofovir-based regimes were mostly associated with low BMD (52.4\%). A statistically significant association between low CD4 count and low BMD was found.

Conclusion Our study concluded that HIV infection is associated with bone loss and low BMD in people living with HIV (PLHIV) irrespective of its treatment with ART. PLHIV are at a greater risk of bone loss secondary to decreased BMD. Among the ART regimens, tenofovir-based regimens are mostly associated with low BMD. Therefore, all HIV patients should be screened by DEXA scan for BMD status, and timely intervention should be started.
\end{abstract}

\section{Introduction}

Human immunodeficiency virus (HIV) is a disease that affects millions of people globally. In India, HIV prevalence is $0.22 \%(0.16-0.30)$ in 2017 in adult populations (age range: $15-49$ years). ${ }^{1}$ Around 21.40 lakh people are living with HIV. ${ }^{1}$ Statewise, HIV incidence per 1,000 uninfected population in 2017 is highest in Mizoram (1.32) followed by Nagaland (0.59) and Manipur (0.58). ${ }^{1}$ Although Manipur has hardly $0.2 \%$ of India's population, it is contributing nearly $8 \%$ of India's total HIV positive cases (highest among the injecting intravenous drug users). HIV has emerged as a new and serious public health problem in Manipur. HIV infection virtually affects every system of the body, including hematologic
DOI https://doi.org/

10.1055/s-0040-1712815

ISSN 2455-7420.
License terms

(ㅇ) (1) $\ominus \circledast$ 
system, central nervous system, respiratory system, cardiovascular system, and skeletal system. With the advent of highly active antiretroviral therapy (HAART), there has been a significant increase in the life expectancy of HIV infected patients; however, long-term effect and complications are also emerging, such as lipodystrophy, insulin resistance, diabetes, dyslipidemia, and alteration in phosphocalcic metabolism. ${ }^{2}$ Alterations in bone metabolism secondary to HIV infections, especially the change in bone mineral density (BMD) in these patients, are being researched with great interest. Low BMD has been reported in many cross-sectional studies involving younger ${ }^{3-8}$ and older ${ }^{9-12}$ HIV-infected individuals secondary to viral effects and inflammatory effects. Low BMD is significantly associated with a high risk of fracture in people living with HIV (PLHIV).${ }^{13}$ Unfortunately, treatment with HAART also causes change in BMD. In one meta-analysis, the prevalence of osteoporosis was three times higher among HIV-infected patients than among HIVnegative controls, especially among those receiving antiretroviral therapy (ART). ${ }^{14}$ Among the ART, tenofovir (tenofovir disoproxil fumarate [TDF]) has proven to be the most widely involved agent in bone demineralization..$^{15}$ Studies clarifying the cause-effect relationship underlying bone loss related to HIV infection are published from the developed world. So far, there are very few studies in India on this association, and no study has been conducted in this part of our country. Therefore, this study sought an accurate estimate of the prevalence of reduced BMD in a cohort of HIV-infected Manipuri patients with or without HAART. Its awareness could be harnessed to prevent the loss of bone density and subsequently negate potential fractures. This necessitated and provided us the impetus to perform this study to measure the change in BMD of HIV patients irrespective of its treatment with HAART.

\section{Materials and Methods}

This cross-sectional study was conducted at the Department of Medicine and ART Centre of Regional institute of Medical Sciences (RIMS), Imphal, India, for a period of 2 years (September 2016 to August 2018). BMD was measured in 50 HIV patients. In patients with low BMD, its correlation with CD4 count (HIV dependent factor) and body mass index ([BMI] HIV independent factor) were studied. This study also examined the change in BMD among the different regimens of HAART. They were divided into two groups: HAART therapy group and therapy-naive group. The therapy group was further divided according to the HAART regimen: first-line therapy group (tenofovir, lamivudine, and efavirenz [TLE]; zidovudine, lamivudine, and nevirapine [ZLN]] and second-line therapy group.

\section{Inclusion Criteria}

HIV-positive patients aged $>18$ years who visited the Medicine outpatient department or the ART Center, RIMS, or were admitted to the medicine ward were included in the study after obtaining informed consent.

\section{Exclusion Criteria}

Patients with other relevant comorbid conditions that can affect BMD were excluded from the study, such as bone disease, chronic metabolic diseases, chronic liver disease, chronic kidney disease, cancer, prolonged bedrest (more than 6 months), and patient on drugs such as corticosteroids, thyroxine, and lithium.

Personal details were recorded in proper performa, including age, sex, BMI, CD4 count, ART regime (first-line or alternative first- or second-line ART) and DEXA (dual-energy X-ray absorptiometry) report. A detailed relevant clinical examination of every patient was also performed. BMD was measured by DEXA scan using the lunar prodigy advance direct digital densitometry. The BMD was measured at the lumbar spine (L1-L4). Other relevant laboratory tests including hemoglobin, creatinine, liver enzymes, and albumin were also performed.

The World Health Organization (WHO) classifies BMD according to the number of standard deviations below the mean BMD for a healthy, young (25-35 years of age) sexand ethnicity-matched reference population (T-score). ${ }^{16}$ For those aged $>50$ years and postmenopausal women, a T-score of less than or equal to-2.5 at the hip or spine is defined as osteoporosis. Osteopenia is defined as a T-score between-1 and-2.49. A T score between 1 and- 0.9 is normal. For those aged $<50$ years, a Z-score of less than or equal to 2.0 is considered to be abnormal. ${ }^{17}$

HAART was defined as a combination of at least three antiretroviral agents that contained two nucleoside reverse transcriptase inhibitors (NRTIs) plus one protease inhibitor (PI) or one non-NRTI. Second-line ART should consist of a ritonavir-boosted PI plus two NRTIs, one of which should be zidovudine (azidothymidine) or TDF, based on what was used in the first-line therapy. Ritonavir-boosted atazanavir or lopinavir/ritonavir is the preferred PI.

Statistical analysis was performed using SPSS Version 20.0 (IBM Corp., Armonk, New York, United States). Mean and standard deviation were calculated for different study variables. Chi-square test and Student's $t$-test (two-tailed, independent) were used to test the association between BMD and HIV infection in PLHIV with selected variables of interest. A $p$-Value of $<0.05$ was considered as statistically significant.

\section{Results}

BMD was evaluated in a total of 50 HIV-infected patients whose baseline characteristics are shown in -Table 1. The mean age of the patients in the study groups was $43.1 \pm 7.2$ years, with the majority aged 41 to 50 years (46\%). Majority of the cases were females (72\%), and males accounted for $28 \%$. Females have lower bone mass with a faster rate of decreasing bone mass as compared with males. ${ }^{18}$ The mean BMI was $22 \pm 4.1$, and differences in BMI in the ART-naive group and the ART group was not statistically significant. Among 50 patients, 11 (22\%) patients were ART-naive and 39 were on HAART. Based on different regimens, 19 (38\%) patients were on TLE, 12 (24\%) were 
Table 1 Summary of baseline characteristics of study patients

\begin{tabular}{|c|c|c|}
\hline Characteristics & Study patients $(N=50), n(\%)$ & Mean \\
\hline \multicolumn{3}{|l|}{ Age $(y)$} \\
\hline $18-30$ & $03(06)$ & \\
\hline $31-40$ & $17(34)$ & \\
\hline $41-50$ & $23(46)$ & 43.1 \\
\hline $51-60$ & $07(14)$ & \\
\hline \multicolumn{3}{|l|}{ Gender } \\
\hline Female & $36(72)$ & \\
\hline Male & $14(28)$ & \\
\hline \multicolumn{3}{|l|}{ BMI } \\
\hline Not on ART & $11(22)$ & 23.24 \\
\hline On ART & $39(78)$ & 22.55 \\
\hline \multicolumn{3}{|c|}{ ART status (mean CD4 count) } \\
\hline Not on ART & $11(22)$ & 276.64 \\
\hline On ART & $39(78)$ & 582.38 \\
\hline \multicolumn{3}{|c|}{ ART duration (in years) } \\
\hline Before initiation & $11(22)$ & 0.981 \\
\hline $0-5$ & $17(34)$ & 1.118 \\
\hline $5-10$ & $12(24)$ & 1.050 \\
\hline $10-15$ & $10(20)$ & 1.031 \\
\hline \multicolumn{3}{|l|}{ ART regimes } \\
\hline ART naive & $11(22)$ & 0.981 \\
\hline TLE & $21(42)$ & 1.057 \\
\hline ZLN & $10(20)$ & 1.059 \\
\hline Second line & $8(16)$ & 1.144 \\
\hline \multicolumn{3}{|l|}{ History of fracture } \\
\hline ART naive & 0 & \\
\hline On ART & 0 & \\
\hline
\end{tabular}

Abbreviations: ART, antiretroviral therapy; BMI, body mass index; TLE, tenofovir, lamivudine, and efavirenz; ZLN, zidovudine, lamivudine, and nevirapine.

on ZLN, and 8 (16\%) were on second-line ART regimen. The mean BMD of PLHIV in our study was 1.05480. In our study, $21(42 \%)$ patients had normal BMD on DEXA scan, whereas 29 had altered BMD changes (-Table 2). Of the 29 patients, 18 (36\%) had osteopenia and $11(22 \%)$ had osteoporosis. ART-naive patients had the lowest BMD among the groups. Among the patients taking ART (with different ART regimens), $52.4 \%$ of patients on TLE have low BMD. Patients on either ZLN or second-line ART had similar reduction in BMD (50\%) (-Table 3).The difference in BMD in ART-naive patients and HIV patients on ART were statistically significant. Of the ART-naive patients, $81.8 \%$ have reduced BMD (either osteopenia or osteoporosis). Regarding the correlation of BMD with CD4 counts, PLHIV on HAART have a higher CD4 count than ART-naive HIV patients. Low CD4 count is associated with low BMD in HIV patients. The association between CD4 count and BMD is statistically significant $(p<0.05)(-$ Table 4$)$.
Table 2 BMD status on DEXA scans of HIV patients with and without ART

\begin{tabular}{|l|l|l|}
\hline \multirow{2}{*}{ HIV patients } & \multicolumn{2}{|c|}{ BMD } \\
\cline { 2 - 3 } & Normal & $\begin{array}{l}\text { Reduced BMD } \\
\text { (osteopenia/ } \\
\text { osteoporosis) }\end{array}$ \\
\hline ART naive & $02(18.2 \%)$ & $09(81.8 \%)$ \\
\hline ART regimens & $10(47.6 \%)$ & $11(52.4 \%)$ \\
\hline TLE & $05(50.0 \%)$ & $05(50.0 \%)$ \\
\hline ZLN & $04(50.0 \%)$ & $04(50.0 \%)$ \\
\hline Second-line ART &
\end{tabular}

Abbreviations: ART, antiretroviral therapy; BMD, bone mineral density; DEXA, dual-energy X-ray absorptiometry; HIV, human immunodeficiency virus; TLE, tenofovir, lamivudine, and efavirenz; ZLN, zidovudine, lamivudine, and nevirapine.

Note: It shows that $81.8 \%$ of ART-naive patients have reduced BMD (either osteopenia or osteoporosis). Among the different ART regimens, $52.4 \%$ of patients on TLE have low BMD. Patients on either ZLN or second-line ART had a similar reduction in BMD (50\%). 
Table 3 Mean BMD in ART-naive HIV patients and those receiving different $A R T$ regimens

\begin{tabular}{|l|l|l|}
\hline ART regimens & Mean BMD & No. of patients \\
\hline ART naive & 0.98127 & 11 \\
\hline TLE & 1.05695 & 21 \\
\hline ZLN & 1.05970 & 10 \\
\hline Second line & 1.14413 & 8 \\
\hline Total & 1.05480 & 50 \\
\hline
\end{tabular}

Abbreviations: ART, antiretroviral therapy; BMD, bone mineral density; HIV, human immunodeficiency virus; TLE, tenofovir, lamivudine, and efavirenz; ZLN, zidovudine, lamivudine, and nevirapine.

Note: Patients receiving TLE had the lowest BMD, whereas patients receiving second-line ART had the highest BMD among the groups. The difference in BMD in ART-naive patients and HIV patients on ART were statistically significant $(p<0.05)$.

\section{Discussion}

Of the many diseases associated with HIV infections, change in bone metabolism is undermined and underdiagnosed. It may be because of the benign course of its natural history and less case fatality rate Altered bone metabolism and HIV infection of the bone lead to reduced bone size, mass, and strength in PLHIV. ${ }^{19}$ Of the many parameters of alteration in bone metabolism, we mainly focused on bone density with respective interpretation based on the standardized WHO BMD score and grading. Many international studies revealed changes in BMD in these people. Borderi et al and Duvivier et al found that HIV infection of osteoblasts is associated with a negative balance of bone remodeling. ${ }^{20,21}$ Decline in BMD following HIV, and its treatment leading to osteopenia and osteoporosis in both male and female patients with HIV ${ }^{22,23}$ as compared with non-HIV controls ${ }^{11,24}$ were reported. In our study, 29 (58\%) patients had reduced BMD. A meta-analysis found that there was a 6.4-fold reduction in BMD value in HIV-positive patients. ${ }^{14}$

Understanding the pathogenesis of bone loss in HIV is as important as its aftereffects. HIV infection targets $\mathrm{T}$ cells, B cells, and monocytes, which modulate several cytokines, causing persistent systemic inflammation. Osteoclastogenic RANKL (receptor activator of nuclear factor-kB ligand) cytokine production is increased because of reaction with HIV glycoprotein (gp) 120 and stimulation by tumor necrosis factor (TNF) $\alpha$ ؟ whereas the bone-sparing osteoprotegerin (OPG) cytokine is decreased. ${ }^{25-27}$ These ultimately result in marked osteoclast and its precursors causing excessive bone resorption, bone loss, and decrease in BMD. ${ }^{28,29}$ RANKL/ OPG markers were found to be positively associated with BMD and T-/Z-score in HIV-positive patients. Another cytokine interferon (IFN gamma) regulates the RANKL receptor through TNF receptor associated factor-6 [TRAF-6]) and leads to decreased osteoclast production. ${ }^{30,31}$ Decreased plasma OPG/RANKL ratio and a plasma RANKL concentration of $>500 \mathrm{pg} / \mathrm{mL}$ in the HIV patients were associated with low BMD. ${ }^{28}$ Moreover, viral proteins also augments alteration in bone metabolism. HIV viral proteins $\mathrm{Vpr}$ (viral protein R) and gp120 stimulate osteoclast activity, whereas p55-gag suppresses osteoblast activity and increases
Table 4 CD4 count in relation to BMD in HIV patients

\begin{tabular}{|l|l|}
\hline DEXA scan & Mean CD4 count, cells/mm ${ }^{3}$ \\
\hline Normal & 674.81 \\
\hline Osteopenia/ osteoporosis & 399.48 \\
\hline
\end{tabular}

Abbreviations: BMD, bone mineral density; DEXA, dual-energy X-ray absorptiometry; HIV, human immunodeficiency virus.

Notes: It shows that a low CD4 count is associated with low BMD in HIV patients.

$p$-Value $<0.05$ was statistically significant.

osteoblast apoptosis. This bone loss may also be because of the functional regulation of osteoclasts due to higher levels of C-C chemokine receptor 5 (CCR 5$)^{32}$

Osteoporosis was seen in $22 \%$ of cases in our study, which was similar to that of $23 \%$ by Bonjoch et al. ${ }^{33}$ The rate was much higher as compared with the study by Bruera et al. ${ }^{3}$ In western population, osteoporosis was found in approximately 10 to $13 \%$ of HIV-positive patients. An osteoporosis rate of $15 \%$ was reported by Brown and Qaqish. ${ }^{14}$ Approximately 3.7-fold higher occurrence of osteoporosis was recorded in PLHIV. ${ }^{14}$ The lowest osteoporotic rate encountered in HIV patients was $12 \%{ }^{34}$ The plausible theory for higher BMD in mainland Indian population could be because of late diagnosis with simultaneous multiple comorbid opportunistic infections. These variables may exaggerate BMD alteration. Ironically, in our state of Manipur, the population is less but with good literacy and education levels, leading to better societal awareness regarding HIV, and thereby PLHIV turnout and reach to medical facilities are much better. This makes early treatment possible with lesser late signs (early stage).

Having said that, still osteopenia was detected in 36\% of the cases in our study, which was comparable with the study of Casado et $a{ }^{35}$ who studied 285 patients and found the prevalence of osteopenia in HIV population to the tune of 36 to $57 \%$ depending on the presence of secondary factors. The rate of osteopenia detected by Brown and Qaqish was $67 \%,{ }^{14}$ whereas Bonjoch et al reported a rate of $47.5 \%{ }^{33}$ A slightly similar value of $42 \%$ was reported by Sharma et al. ${ }^{34}$

Comparison-wise, a milder form of BMD loss, that is, osteopenia, was more common finding than osteoporosis. ${ }^{34}$ Osteopenia progresses to osteoporosis in approximately $12 \%$ of HIV patients. ${ }^{34}$ In our study too, osteopenia was more commonly detected than osteoporosis. Similar results were reported by Cazanave et al as well..$^{22}$ They found that more than half the HIV-infected patients had osteopenia and one-third had more severe bone loss, that is, osteoporosis. ${ }^{22}$ McComsey et al also reported similar results. ${ }^{15}$ These findings suggest that although PLHIV patients were prone to bone mineral loss, the magnitude of loss is less and subjects to several variables.

HIV severity (with respect to CD4 count) also contributes to reduced BMD. ${ }^{36-38}$ Greater bone loss and BMD were found in HIV-infected individuals with a low CD4 cell count $\left(<50\right.$ cells $\left./ \mathrm{mm}^{3}\right)$ than those with a higher CD4 cell count $\left(>500\right.$ cells $\left./ \mathrm{mm}^{3}\right) \cdot{ }^{39}$ In our study, we found that a reduction in BMD in HIV patients is associated with low CD4 count and is statistically significant. HIV patients with normal 
BMD in our study had a higher CD4 count (mean CD4 count: 674.81 cells $/ \mathrm{mm}^{3}$ ) compared with patients having low BMD (mean CD4 count: 399.48 cells $/ \mathrm{mm}^{3}$ ). Rivas et al also found a similar association between CD4 and BMD. ${ }^{23}$

Meanwhile, there were reports of fracture risk in PLHIV, ${ }^{13}$ with higher rates of approximately 30 to 70\% than HIVnegative patients. ${ }^{15}$ In our study, fracture was not reported in any of the patients within the follow-up of 3 years possibly due to better health awareness.

Another important aspect was the treatment of HIV itself causing osteopenia and osteoporosis. All ART regimens at their initiation stages decrease BMD irrespective of the regimen. According to Knobel et al, ${ }^{6}$ both osteopenia and osteoporosis were reported in patients on HAART treatment and in therapy-naive patients, with a similar percentage of osteopenia and osteoporosis.

Among the patients on various HAART regimens in our study, $52.4 \%$ on TLE had low BMD. Patients on either ZLN or second-line ART had a similar reduction in BMD (50\%). Specifically, tenofovir-based regimen causes more bone loss (1-3\% greater bone mineral loss) as compare with others. ${ }^{40-42}$ BMD in the spine and hip were showing more decrease values with tenofovir than with other regimens. ${ }^{42}$ Similar findings were shared by Haskelberg et al as well. ${ }^{43}$ Biochemically, TDF is converted to tenofovir through di-ester hydrolysis in plasma. Selective uptake of tenofovir by osteoclasts causes cellular stress, which alters gene expression involved in signaling osteoblast activity, and this results in decreased bone formation. ${ }^{44}$ It also causes bone demineralization and loss of renal calcium and phosphate with compensatory reabsorption. Ironically, in our country, TDF-based regimen was often started as an initial first-line therapy for HIV treatment, unless contraindicated. For those patients on alternative first- or second-line ART having low BMD, TDF might have been used initially for a variable duration before switching to the latter. Therefore, contribution of TDF to low BMD, even in the aforementioned patients, was quite a possibility. Other ART drugs causing low BMD were PIs and NRTIs. Mechanism of PIs causes a reduction in the production of the active form of vitamin $\mathrm{D} 1,25(\mathrm{OH}) 2 \mathrm{D}$ by inhibiting 25-hydroxylase and $1-\alpha$ hydroxylase, resulting in a decrease in calcium absorption and decrease in bone density. Also, PIs increase osteoclast activity through proteasomal degradation of TRAF- 6 , leading to unblocking of RANKL signaling. PI also inhibits osteoblastogenesis. NRTIs, on the other hand, inhibit the DNA polymerase, thereby inhibiting the replication of mitochondrial DNA and causing mitochondrial damage. It leads to lactic academia and causes osteopenia. Acidosis is buffered by bone calcium hydroxyapatite loss, resulting in osteoporosis. ${ }^{15}$

It is worth mentioning that our study showed $81.8 \%$ of ART-naive patients having reduced BMD (either osteopenia or osteoporosis). This finding could be corroborated with that of the study by Kruger and Nell, ${ }^{45}$ who also suggested a high prevalence of osteopenia and osteoporosis in HAART-naive patients. Such findings could be explained by the fact that sustained HIV viremia (unchecked) results in chronic activation of affected host immune cells causing inflammation and bone remodeling. Also, viral proteins activation could result in similar mechanism cascade. ${ }^{45}$

Duration of such ART was also another important factor in reducing BMD. Decline in BMD was reported with prolonged continuous course of ART according to Grund et al, ${ }^{46}$ who evaluated 214 patients and observed that BMD in patients on continuous ART declined per year by $0.8 \%$ (hip), $0.4 \%$ (spine DEXA), and $2.4 \%$ (spine-quantitative CT [computed tomography]). Few studies reported a 2 to 6\% drop in BMD within the initial 2 years after initiation of various ART regimens. ${ }^{15}$ However, in our study, the differences in BMD in relation to the duration of ART are not statistically significant.

\section{Limitations}

This study has some limitations. First, the small sample size of the study with no controls may influence the results. Second, the cross-sectional design of the study limits the extension of its interpretation to the causality of associations. Third, all the patients were from the same examination center and were not compared with ART patients from other centers or states, and therefore selection bias could not be excluded. Fourth, HIV viral load was not assessed because of limited resources. Despite these limitations, our study has the advantage of being the first study evaluating bone health in HIV patients in this area cohort.

\section{Conclusion}

We conclude that PLHIV are at a greater risk of bone loss. Among other factors, HIV itself and treatment with HAART (especially tenofovir) also contribute to reduction in BMD. Low BMI, advanced stage of disease, and low CD4 counts are associated with low bone mass in HIV-positive individuals. Low BMD increases the risk of potential fracture, thereby necessitating preventive measures. Further longitudinal studies involving a large population are required to obtain more conclusive results and to establish a causal association between HIV infection and bone loss. Therefore, we recommend screening of all HIV patients by DEXA scan for BMD status and timely intervention.

\section{Conflict of Interest}

None declared.

\section{References}

1 National AIDS Control Organization \& ICMR-National Institute of Medical Statistics, HIV Estimations 2017: Technical Report. New Delhi: NACO, Ministry of Health and Family Welfare, Government of India; 2018

2 Grafodatskaya D, Cytrynbaum C, Weksberg R. The health risks of ART. EMBO Rep 2013;14(2):129-135

3 Bruera D, Luna N, David DO, Bergoglio LM, Zamudio J. Decreased bone mineral density in HIV-infected patients is independent of antiretroviral therapy. AIDS 2003;17(13):1917-1923

4 Carr A, Miller J, Eisman JA, Cooper DA. Osteopenia in HIVinfected men: association with asymptomatic lactic acidemia and lower weight pre-antiretroviral therapy. AIDS 2001;15(6):703-709 
5 Dolan SE, Huang JS, Killilea KM, Sullivan MP, Aliabadi N, Grinspoon S. Reduced bone density in HIV-infected women. AIDS 2004;18(3):475-483

6 Knobel H, Guelar A, Vallecillo G, Nogués X, Díez A. Osteopenia in HIV-infected patients: is it the disease or is it the treatment? AIDS 2001;15(6):807-808

7 Tebas P, Powderly WG, Claxton S, et al. Accelerated bone mineral loss in HIV-infected patients receiving potent antiretroviral therapy. AIDS 2000;14(4):F63-F67

8 Teichmann J, Stephan E, Lange U, et al. Osteopenia in HIVinfected women prior to highly active antiretroviral therapy. J Infect 2003;46(4):221-227

9 Arnsten JH, Freeman R, Howard AA, Floris-Moore M, Lo Y, Klein RS. Decreased bone mineral density and increased fracture risk in aging men with or at risk for HIV infection. AIDS 2007;21(5):617-623

10 Arnsten JH, Freeman R, Howard AA, Floris-Moore M, Santoro $\mathrm{N}$, Schoenbaum EE. HIV infection and bone mineral density in middle-aged women. Clin Infect Dis 2006;42(7):1014-1020

11 Jones S, Restrepo D, Kasowitz A, et al. Risk factors for decreased bone density and effects of HIV on bone in the elderly. Osteoporos Int 2008;19(7):913-918

12 Yin M, Dobkin J, Brudney K, et al. Bone mass and mineral metabolism in HIV+ postmenopausal women. Osteoporos Int 2005; 16(11):1345-1352

13 Triant VA, Brown TT, Lee H, Grinspoon SK. Fracture prevalence among human immunodeficiency virus (HIV)-infected versus non-HIV-infected patients in a large U.S. healthcare system. J Clin Endocrinol Metab 2008;93(9):3499-3504

14 Brown TT, Qaqish RB. Antiretroviral therapy and the prevalence of osteopenia and osteoporosis: a meta-analytic review. AIDS 2006;20(17):2165-2174

15 McComsey GA, Tebas P, Shane E, et al. Bone disease in HIV infection: a practical review and recommendations for HIV care providers. Clin Infect Dis 2010;51(8):937-946

16 Report of a WHO Study Group. Assessment of fracture risk and its application to screening for postmenopausal osteoporosis. World Health Organ Tech Rep Ser 1994;843:1-129

17 National Osteoporosis Foundation. Clinician's Guide to Prevention and Treatment of Osteoporosis. Washington, DC: National Osteoporosis Foundation; 2010

18 WHO Scientific Group, Prevention and Management of Osteoporosis Geneva: World Health Organization; 2003

19 Bolland MJ, Grey A. HIV and low bone density: responsible party, or guilty by association? IBMS boneKEy 2011;8:7-15

20 Borderi M, Gibellini D, Vescini F, et al. Metabolic bone disease in HIV infection. AIDS 2009;23(11):1297-1310

21 Duvivier C, Kolta S, Assoumou L, et al. Greater decrease in bone mineral density with protease inhibitor regimens in HIV-1 infected naïve patients. AIDS 2009;23:817-824

22 Cazanave C, Dupon M, Lavignolle-Aurillac V, et al; Groupe d'Epidémiologie Clinique du SIDA en Aquitaine. Reduced bone mineral density in HIV-infected patients: prevalence and associated factors. AIDS 2008;22(3):395-402

23 Rivas P, Górgolas M, García-Delgado R, Díaz-Curiel M, Goyenechea A, Fernández-Guerrero ML. Evolution of bone mineral density in AIDS patients on treatment with zidovudine/lamivudine plus abacavir or lopinavir/ritonavir. HIV Med 2008;9(2):89-95

24 Dolan SE, Carpenter S, Grinspoon S. Effects of weight, body composition, and testosterone on bone mineral density in HIV-infected women. J Acquir Immune Defic Syndr 2007;45(2):161-167

25 Vikulina T, Fan X, Yamaguchi M, et al. Alterations in the immuno-skeletal interface drive bone destruction in HIV-1 transgenic rats. Proc Natl Acad Sci U S A 2010;107(31):13848-13853

26 Moir S, Ho J, Malaspina A, et al. Evidence for HIV-associated $B$ cell exhaustion in a dysfunctional memory B cell compartment in HIV-infected viremic individuals. J Exp Med 2008;205(8):1797-1805

27 Hittinger G, Poggi C, Delbeke E, Profizi N, Lafeuillade A. Correlation between plasma levels of cytokines and HIV-1 RNA copy number in HIV-infected patients. Infection 1998;26(2):100-103

28 Gibellini D, Borderi M, De Crignis E, et al. RANKL/OPG/TRAIL plasma levels and bone mass loss evaluation in antiretroviral naive HIV-1-positive men. J Med Virol 2007;79(10):1446-1454

29 Ueland T, Bollerslev J, Godang K, Müller F, Frøland SS, Aukrust P. Increased serum osteoprotegerin in disorders characterized by persistent immune activation or glucocorticoid excess-possible role in bone homeostasis. Eur J Endocrinol 2001;145(6):685-690

30 Gao Y, Grassi F, Ryan MR, et al. IFN-gamma stimulates osteoclast formation and bone loss in vivo via antigen-driven $\mathrm{T}$ cell activation. J Clin Invest 2007;117(1):122-132

31 Takayanagi H, Ogasawara K, Hida S, et al. T-cell-mediated regulation of osteoclastogenesis by signalling cross-talk between RANKL and IFN-Y. Nature 2000;408(6812) :600-605

32 Lee JW, Hoshino A, Inoue K, et al. The HIV co-receptor CCR5 regulates osteoclast function. Nat Commun 2017;8(1):2226

33 Bonjoch A, Figueras M, Estany C, et al; Osteoporosis Study Group. High prevalence of and progression to low bone mineral density in HIV-infected patients: a longitudinal cohort study. AIDS 2010;24(18):2827-2833

34 Sharma A, Flom PL, Weedon J, Klein RS. Prospective study of bone mineral density changes in aging men with or at risk for HIV infection. AIDS 2010;24(15):2337-2345

35 Casado JL, Bañon S, Andrés R, Perez-Elías MJ, Moreno A, Moreno S. Prevalence of causes of secondary osteoporosis and contribution to lower bone mineral density in HIV-infected patients. Osteoporos Int 2014;25(3):1071-1079

36 Ofotokun I, McIntosh E, Weitzmann MN. HIV: inflammation and bone. Curr HIV/AIDS Rep 2012;9(1):16-25

37 Ofotokun I, Titanji K, Vunnava A, et al. Antiretroviral therapy induces a rapid increase in bone resorption that is positively associated with the magnitude of immune reconstitution in HIV infection. AIDS 2016;30(3):405-414

38 Battalora LA, Young B, Overton ET. Bone, fractures, antiretroviral therapy and HIV. Curr Infect Dis Rep 2014;16(2):393

39 Grant PM, Kitch D, McComsey GA, et al. Low baseline CD4+ count is associated with greater bone mineral density loss after antiretroviral therapy initiation. Clin Infect Dis 2013;57(10):1483-1488

40 Brown TT, Hoy J, Borderi M, et al. Recommendations for evaluation and management of bone disease in HIV. Clin Infect Dis 2015;60(8):1242-1251

41 McComsey GA, Kitch D, Daar ES, et al. Bone mineral density and fractures in antiretroviral-naive persons randomized to receive abacavir-lamivudine or tenofovir disoproxil fumarate-emtricitabine along with efavirenz or atazanavir-ritonavir: Aids Clinical Trials Group A5224s, a substudy of ACTG A5202. J Infect Dis 2011;203(12):1791-1801

42 Grant PM, Cotter AG. Tenofovir and bone health. Curr Opin HIV AIDS 2016;11(3):326-332

43 Haskelberg H, Hoy JF, Amin J, Ebeling PR, Emery S, Carr A; STEAL Study Group. Changes in bone turnover and bone loss in HIV-infected patients changing treatment to tenofovir-emtricitabine or abacavir-lamivudine. PLoS One 2012;7(6):e38377

44 Mulubwa M, Viljoen M, Kruger I, Kruger H, Rheeders M. Bone turnover markers in HIV-infected women on tenofovir-based antiretroviral therapy. South Afr J HIV Med 2017;18(1):a739

45 Kruger MJ, Nell TA. Bone mineral density in people living with HIV: a narrative review of the literature. AIDS Res Ther 2017;14(1):35

46 Grund B, Peng G, Gibert CL, et al; INSIGHT SMART Body Composition Substudy Group. Continuous antiretroviral therapy decreases bone mineral density. AIDS 2009;23(12):1519-1529 